\title{
Fatores associados ao surgimento de ansiedade/depressão em policiais militares: uma revisão integrativa
}

Factors associated with the emergence of anxiety/depression in military police officers: an integrative review

Factores asociados a la aparición de ansiedad / depresión en policías militares: una revisión integradora

Karen Laís Azevêdo Oliveira Sousa

ORCID: https://orcid.org/0000-0002-0308-417X Centro Universitário de Ciências e Tecnologia do Maranhão, Brasil E-mail: Karen.lais05@gmail.com

Hálmisson D’Árley Santos Siqueira

ORCID: https://orcid.org/0000-0001-9831-5892 Centro Universitário de Ciências e Tecnologia do Maranhão, Brasil E-mail: halmisson@yahoo.com.br Wenderson Costa da Silva ORCID: https://orcid.org/0000-0001-6031-9775 Universidade Federal do Maranhão, Brasil E-mail: wendersoncosta09@hotmail.com

Francisléia Falcão França Santos Siqueira ORCID: https://orcid.org/0000-0001-8783-5139 Universidade Estadual do Maranhão, Brasil E-mail: gpmsaude@gmail.com

Solygardia Albuquerque Maciel Teixeira ORCID: https://orcid.org/0000-0003-0214-0982 Centro Universitário Nossa Senhora do Patrocínio, Brasil E-mail: solygardia@bol.com.br

Thalia Jeovana da Silva Pereira ORCID: https://orcid.org/0000-0003-0323-5318 Centro Universitário de Ciências e Tecnologia do Maranhão, Brasil E-mail: thaliajeovana96@gmail.com

Marta Valeria Soares Chaves ORCID: https://orcid.org/0000-0002-3797-3696 Centro Universitário de Ciências e Tecnologia do Maranhão, Brasil E-mail: martavaleria706@gmail.com

Ana Tereza Santos Dias de Almeida ORCID: https://orcid.org/0000-0001-9927-1836 Centro Universitário de Ciências e Tecnologia do Maranhão, Brasil E-mail: aterezadias@gmail.com

Juliana Helen Almeida de Lima ORCID: https://orcid.org/0000-0002-1867-6450 Centro Universitário de Ciências e Tecnologia do Maranhão, Brasil E-mail: jhelenlima22@gmail.com

Rayssa Stefani Cesar Lima ORCID: https://orcid.org/0000-0001-8859-7961

Universidade Estadual do Maranhão, Brasil E-mail: rayssastefany13@hotmail.com

Raimundo Nonato de Sousa Nunes Queiroz ORCID: https://orcid.org/0000-0002-0888-757X Centro Universitário de Ciências e Tecnologia do Maranhão, Brasil E-mail: raytimbiras@hotmail.com

Leonardo Carneiro Vieira

ORCID: https://orcid.org/0000-0002-5192-9018 Centro Universitário de Ciências e Tecnologia do Maranhão, Brasil E-mail: leonardo.viera06@hotmail.com

Zaira Arthemisa Mesquita Araújo ORCID: https://orcid.org/0000-0002-6625-034X Pontifícia Universidade Católica de Minas Gerais, Brasil E-mail: Zaira.psicologia@ hotmail.com 


\author{
Antônio Cássio Vaz \\ ORCID: https://orcid.org/0000-0002-1368-3176 \\ Pontifícia Universidade Católica de Minas Gerais, Brasil \\ Email: psicologoacv@gmail.com \\ Linccon Fricks Hernandes \\ ORCID: https://orcid.org/0000-0002-7642-3080 \\ Escola Superior de Ciências da Santa Casa de Misericórdia de Vitória, Brasil \\ E-mail: fricksjr@hotmail.com \\ Brunna Matos Sousa \\ ORCID: https://orcid.org/0000-0002-7009-6488 \\ Centro Universitário de Ciências e Tecnologia do Maranhão, Brasil \\ E-mail:brunnamattos4@gmail.com
}

\begin{abstract}
Resumo
O trabalho do policial militar pode implicar no aparecimento de transtornos ansiedade. Podendo ter impactos significativos na saúde do policial, afetando sua qualidade de vida e as relações sociais, podem ainda prejudicar o desempenho quanto às suas atribuições e atuações de serviço e colocar em risco sua vida e a de terceiros. O presente estudo objetivou analisar, por meio da literatura, as evidências científicas dos fatores associados ao surgimento de ansiedade/depressão em policiais militares. Tratou-se de uma revisão integrativa de literatura, para qual se formulou a seguinte questão clínica: "Quais as evidências científicas sobre os fatores associados ao surgimento de ansiedade/depressão em policiais militares?”. As bases de dados foram consultadas, por meio de descritores e palavras-chave, entre os anos de 2015 a 2020, nos idiomas português, espanhol e inglês. Desse modo, treze estudos foram incluídos nesta revisão, observou-se que os policiais militares relataram sofrer por ansiedade/depressão variaram de 19 a 50 anos de idade, prevaleceu o sexo feminino sendo mais vulneráveis aos transtornos, foi identificado que os policiais do setor administrativo e processos operacionais burocráticos, são menos propensos a desenvolver transtornos de ansiedade ou um quadro patológico de depressão, a convivência com a violência, o constante risco de morte e as cargas excessivas de trabalho são fatores que causam estresse, ansiedade e podem desencadear a depressão afetando de forma significativa a qualidade de vida desses policiais. O que permite concluir que todos os resultados relataram a necessidade de otimizar o ambiente de trabalho através da conscientização promovendo conhecimento adequado sobre transtornos emocionais que são rotineiros, tornando possível uma redução nos índices de adoecimento dessa categoria.
\end{abstract}

Palavras-chave: Polícia; Ansiedade; Depressão; Trabalho.

\begin{abstract}
The work of a military police officer can lead to the appearance of anxiety disorders. They can have significant impacts on police officers' health, affecting their quality of life and social relationships, and can also affect their performance in terms of their duties and service activities and put their lives and that of third parties at risk. The present study aimed to analyze, through the literature, the scientific evidence of the factors associated with the emergence of anxiety/depression in military police officers. It was an integrative literature review, for which the following clinical question was formulated: "What is the scientific evidence on the factors associated with the emergence of anxiety/depression in military police officers?". The databases were consulted, using descriptors and keywords, between the years 2015 to 2020, in Portuguese, Spanish and English. Thus, thirteen studies were included in this review, it was observed that military police officers reported suffering from anxiety/depression ranged from 19 to 50 years of age, females prevailed being more vulnerable to disorders, it was identified that police officers from the administrative sector and bureaucratic operational processes, are less likely to develop anxiety disorders or a pathological condition of depression, living with violence, the constant risk of death and excessive workloads are factors that cause stress, anxiety and can trigger depression affecting significantly the quality of life of these officers. Which allows us to conclude that all the results reported the need to optimize the work environment through awareness, promoting adequate knowledge about emotional disorders that are routine, making it possible to reduce the rates of illness in this category.
\end{abstract}

Keywords: Police; Anxiety; Depression; Work.

\title{
Resumen
}

El trabajo de un policía militar puede provocar la aparición de trastornos de ansiedad. Pueden tener impactos significativos en la salud de los policías, afectando su calidad de vida y relaciones sociales, y también pueden afectar su desempeño en cuanto a sus funciones y actividades de servicio y poner en riesgo su vida y la de terceros. El presente estudio tuvo como objetivo analizar, a través de la literatura, la evidencia científica de los factores asociados a la aparición de ansiedad / depresión en policías militares. Se trató de una revisión integradora de la literatura, para lo cual se formuló la siguiente pregunta clínica: “¿Cuál es la evidencia científica sobre los factores asociados a la aparición de ansiedad / depresión en policías militares?". Se consultaron las bases de datos, utilizando descriptores y palabras clave, entre los años 2015 a 2020, en portugués, español e inglés. Así, en esta revisión se incluyeron trece estudios, se observó que los policías militares reportaron padecer ansiedad / depresión en el rango de 19 a 50 años de 
edad, prevalecieron las mujeres siendo más vulnerables a los trastornos, se identificó que los policías del sector administrativo y procesos operativos burocráticos, tienen menos probabilidades de desarrollar trastornos de ansiedad o una condición patológica de depresión, convivir con la violencia, el riesgo constante de muerte y las cargas laborales excesivas son factores que causan estrés, ansiedad y pueden desencadenar depresión afectando significativamente la calidad de vida de estos oficiales. Lo que nos permite concluir que todos los resultados informaron la necesidad de optimizar el clima laboral a través de la concientización, promoviendo un conocimiento adecuado sobre los trastornos emocionales que son rutinarios, permitiendo reducir las tasas de enfermedad en esta categoría.

Palabras clave: Policía; Ansiedad; Depresión; Trabajo.

\section{Introdução}

A prática policial movimenta-se para além da técnica e pode carregar consigo a rotina de situações de sofrimento e angústias. A atividade militar não se resume ao serviço diário, a profissão implica em constante estado de alerta e atenção redobrada, mesmo quando o profissional está em momento de descanso. No trabalho do policial militar, a morte está presente em uma frequência maior do que se comparado às demais categorias profissionais, seja a morte das vítimas, dos criminosos, ou dos próprios companheiros de trabalho, exigindo que eles tenham que lidar com a ameaça da própria vida mais frequentemente, o que pode implicar no aparecimento de transtornos de ansiedade e depressivos (Oliveira \& Santos, 2010).

No ano de 2010, foi realizada uma pesquisa para verificar as fontes de estresse dos policiais militares. Os principais eventos estressores mencionados foram; com 81,8\%, a necessidade de realizar hora-extra para agregar ao salário e, com 59,6\%, o fato de trabalhar aos finais de semana. Tais fatores são determinantes para o aparecimento de transtornos psiquiátricos de naturezas distintas, além de poderem, ainda, prejudicar o desempenho quanto às suas atribuições e atuações em serviço e colocar em risco a sua vida ou a vida de terceiros (Souza, Minayo, Silva, \& Pires, 2012).

A ansiedade é um estado emocional natural que acompanha o ser humano em sua existência. A ansiedade normal pode ser considerada um sinal de alerta que proporciona ao indivíduo permanecer atento, concentrado ou vigilante, tendo como base objetiva uma ameaça ou perigo existente e decorrente da realidade externa, tornando-se, assim, um mecanismo fundamental para a sua sobrevivência. A ansiedade patológica é distinguida pela intensidade e o caráter anacrônico, constante e desproporcional ao ambiente ou estímulo, caracterizando-se por um sentimento desagradável de assimilação negativa em relação ao futuro (Guimarães et al., 2015).

O termo depressão, por sua vez, designa um estado afetivo aparentemente normal, um sintoma, uma síndrome ou várias doenças. A depressão tem sido caracterizada como episódio patológico no qual existe perda de interesse ou prazer, distúrbios do sono e apetite, retardo motor, sentimentos de inutilidade ou culpa, distúrbios cognitivos e diminuição da energia. A pessoa diagnosticada com a patologia age e reage a situações corriqueiras com desânimo e apresenta características tendenciosas ao suicídio e frequente pensamento em morte (Apóstolo, Figueiredo, Mendes, \& Rodrigues, 2011).

Devido à procura insuficiente pelo cuidado dos fenômenos emocionais relacionados aos profissionais da segurança pública ostensiva, considera-se que estudar os determinantes psicossociais que aumentam o risco de ansiedade e depressão em meio à realidade dos policiais militares é fundamental para alcançar uma melhor compreensão sobre a saúde mental da categoria, tendo em vista esta como sendo uma das de maior convivência com fatores de estresse. Assim, pretende-se, com os resultados obtidos por este estudo, apontar melhorias a serem aplicadas tanto na formação quanto na educação permanente desses profissionais.

A promoção de saúde mental no trabalho dos policiais pode ser uma estratégia para aliviar o desgaste mental, a ansiedade, o estresse e a insegurança frente ao ofício de sua carreira, melhorando a qualidade de vida dos trabalhadores. A partir daí, pode-se alcançar uma maior disposição em seu setor de trabalho, o que tornará o profissional mais ativo e participativo socialmente e, consequentemente, menos ansioso e depressivo, com o reconhecimento dos fatores de risco frente às situações estressantes em seu cotidiano. 
Este estudo almeja propiciar um conhecimento adequado dos transtornos emocionais que são rotineiros em seu trabalho. Os resultados obtidos podem fornecer subsídios para que ações de suporte e prevenção em saúde do Policial Militar sejam realizadas continuamente, tornando possível uma redução nos índices de adoecimento dessa categoria, buscando, consequentemente, resultados mais eficazes no combate a esses transtornos de origem emocional.

Com base no exposto, traçou-se a seguinte problemática: quais as evidências científicas sobre os fatores associados ao surgimento de ansiedade/depressão em policiais militares?

O objetivo geral desta pesquisa foi analisar, por meio da literatura, as evidências científicas dos fatores associados ao surgimento de ansiedade/depressão em policiais militares. Especificamente, objetivou-se: identificar dados sociodemográficos que envolvem os policiais com quadro de ansiedade/depressão; identificar os determinantes psicossociais que aumentam o risco de ansiedade e depressão entre os policiais militares; e determinar de que forma o quadro de ansiedade/depressão afeta a qualidade de vida desses policiais.

\section{Metodologia}

Trata-se de um estudo de pesquisa bibliográfica do tipo revisão integrativa da literatura. Esse procedimento foi escolhido por possibilitar a síntese e análise do conhecimento científico já produzido sobre o tema "FATORES ASSOCIADOS AO SURGIMENTO DE ANSIEDADE/DEPRESSÃO EM POLICIAIS MILITARES: uma revisão integrativa”. Esta revisão utilizou a metodologia proposta no estudo de Oliveira et al. (2016).

De acordo com Ercole, Melo e Alcoforado (2014), a revisão integrativa de literatura é um método que tem como finalidade sintetizar resultados obtidos em pesquisas de maneira sistemática, ordenada e abrangente, mediante diferentes metodologias. É denominada integrativa porque fornece informações mais amplas sobre um assunto, constituindo um corpo de conhecimento e podendo ser direcionada para a definição de conceitos, revisão de teorias ou análise metodológica dos estudos. Esse método proporciona a combinação de dados da literatura teórica e empírica, proporcionando maior compreensão do tema de interesse.

O tema determinou a construção da estratégia PICO, que representa um acrônimo para Paciente (P), Interesse (I), Contexto (Co), a qual foi utilizada para a geração da questão norteadora desta revisão integrativa da literatura: "Quais as evidências científicas sobre os fatores associados ao surgimento de ansiedade e depressão em policiais militares?"

Para a localização dos estudos relevantes que respondessem à pergunta de pesquisa, utilizou-se de descritores indexados e não indexados (palavras-chave) nos idiomas português, inglês e espanhol. Os descritores foram obtidos a partir do Medical Subject Headings (MESH), dos Descritores em Ciências da Saúde (DeCS) e dos títulos CINAHL, como mostra o Quadro 1.

Consultaram-se, por meio de descritores e palavras-chave, as bases de dados PubMed da National Library of Medicine; BVS (Biblioteca Virtual da Saúde), coordenada pela BIREME e composta de bases de dados produzidas pela Rede BVS, como LILACS, além da base de dados Medline e outros tipos de fontes de informação; e CINAHL (Cumulative Index to Nursing and Allied Health Literature). 
Quadro 1. Elementos da estratégia PICo, descritores e palavras-chave utilizados. Caxias, MA, Brasil, 2020.

\begin{tabular}{|c|c|c|c|c|c|}
\hline \multicolumn{2}{|r|}{ Elementos } & Mesh & Decs & Títulos cinahl & Palavras-chave \\
\hline $\mathrm{P}$ & "Policiais" & "Police" & $\begin{array}{l}\text { "Police" } \\
\text { "Policia" } \\
\text { "Policia" }\end{array}$ & "Police" & "Policia" \\
\hline I & $\begin{array}{l}\text { "Ansiedade e } \\
\text { Depressão" }\end{array}$ & $\begin{array}{c}\text { "Anxiety" } \\
\text { "Depression" }\end{array}$ & $\begin{array}{l}\text { "Anxiety" } \\
\text { "Ansiedad" } \\
\text { "Ansiedade" } \\
\text { "Depression" } \\
\text { "Depressión" } \\
\text { "Depressão" }\end{array}$ & $\begin{array}{l}\text { "Anxiety” } \\
\text { "Depression" }\end{array}$ & $\begin{array}{l}\text { "Ansiedade" } \\
\text { “Depressão" }\end{array}$ \\
\hline $\mathrm{Co}$ & "Trabalho" & "work" & $\begin{array}{c}\text { “Work” } \\
\text { "Trabajo" } \\
\text { "Trabalho", }\end{array}$ & "Work" & "Trabalho" \\
\hline
\end{tabular}

Fonte: Descritores, Títulos e Palavras-chaves (2020).

Os termos utilizados durante a pesquisa foram classificados e combinados nos bancos de dados, resultando em estratégias específicas, em que a estratégia de busca empregada foi a booleana com operador lógico de pesquisa AND.

Como critérios de inclusão, utilizaram-se estudos disponíveis em sua totalidade, publicados nos últimos seis anos, de 2015 até 2020, nos idiomas Português, Espanhol e Inglês. Foram excluídos da busca inicial capítulos de livros, resumos, textos incompletos, teses, dissertações, monografias, relatos técnicos e outras formas de publicação que não artigos científicos completos. Por fim, a análise para seleção dos estudos foi realizada em duas fases.

Na primeira fase, os estudos foram pré-selecionados segundo os critérios de inclusão e exclusão e de acordo com a estratégia de busca em cada base de dados.

Encontraram-se dezoito (18) estudos como busca geral na BVS, sendo que, limitando a busca para artigos com texto completo realizado com humanos nos últimos seis anos, obtiveram-se oito (8) estudos; destes, foram analisados títulos e resumos, dentre os quais apenas cinco (5) estudos foram condizentes com a questão desta pesquisa.

Na base PUBMED, como busca total foram encontrados cento e trinta e sete (137) estudos. Aplicando na pesquisa o filtro que limita por texto completo dos últimos seis anos com humanos, reduziu-se para cinquenta e um (51) estudos; destes, foram analisados títulos e resumos e teve-se como resultado final sete (7) estudos.

$\mathrm{Na}$ base CINAHL, como busca total, foram encontrados cento e quarenta e quatro (144) estudos. Aplicando na pesquisa o filtro que limita por texto completo dos últimos seis anos com humanos, foram obtidos vinte e oito (28) estudos; destes, foram analisados títulos e resumos e teve-se como resultado final um (1) estudo.

Correspondendo à segunda fase da análise para a seleção dos artigos, os mesmos foram avaliados quanto ao potencial de participação no estudo, observando o atendimento à questão de pesquisa, bem como o tipo de investigação, objetivos, amostra, método, desfechos, resultados e conclusão, resultando em um número final de treze (13) artigos.

Com relação à análise e interpretação dos resultados, nesta etapa foram analisadas as informações coletadas nos artigos científicos e criadas categorias analíticas que facilitaram a ordenação e a sumarização de cada estudo. Essa categorização foi realizada de forma descritiva, indicando os dados mais relevantes para o estudo.

A pesquisa levou em consideração os aspectos éticos quanto às citações dos estudos, respeitando a autoria das ideias, os conceitos e as definições presentes nos artigos incluídos na revisão.

Por fim, optou-se pela análise em forma estatística e de texto, utilizando cálculos matemáticos e inferências, que serão apresentados em quadros e tabelas para facilitar a visualização e compreensão. 


\section{Resultados}

A apresentação dos resultados está organizada em duas partes. A primeira está relacionada com a caracterização dos estudos, já a segunda, relaciona-se ao cumprimento do objetivo do estudo, que diz respeito a fatores associados ao surgimento de ansiedade/depressão entre os policiais militares.

Dos treze (13) estudos nesta revisão, todos estavam na língua inglesa (100\%). A maioria das publicações foram concentradas nos anos de 2015, 2016 e 2019 (23,08\%) com abordagem quantitativa (92,31\%). O nível de evidência predominante foi seis (estudos transversais); os Estados Unidos e Canadá foram os países com mais estudos incluídos $(23,08 \%)$, conforme demonstrado na Tabela 1.

Tabela 1. Análise descritiva das produções científicas acerca dos fatores associados ao surgimento de ansiedade/depressão entre os policiais militares. Caxias, MA, Brasil, 2020. ( $\mathrm{n}=13)$.

\begin{tabular}{|c|c|c|}
\hline VARIAVEIS & $\mathbf{N}$ & $\%$ \\
\hline \multicolumn{3}{|l|}{ Base de dados } \\
\hline CINAHL & 1 & 7,69 \\
\hline BIREME & 5 & 38,46 \\
\hline PUBMED & 7 & 53,85 \\
\hline \multicolumn{3}{|l|}{ Abordagem do estudo } \\
\hline Quantitativo & 12 & 92,31 \\
\hline Qualitativo & 1 & 7,69 \\
\hline \multicolumn{3}{|l|}{ Ano } \\
\hline 2015 & 3 & 23,08 \\
\hline 2016 & 3 & 23,08 \\
\hline 2017 & 1 & 7,69 \\
\hline 2018 & 2 & 15,38 \\
\hline 2019 & 3 & 23,08 \\
\hline 2020 & 1 & $\begin{array}{l}20,60 \\
7,69\end{array}$ \\
\hline \multicolumn{3}{|l|}{ Idiomas } \\
\hline Inglês & 13 & 100,0 \\
\hline \multicolumn{3}{|l|}{ País } \\
\hline Canadá & 3 & 23,08 \\
\hline China & 1 & 7,69 \\
\hline União Europeia & 1 & 7,69 \\
\hline Sri Lanka & 1 & 7,69 \\
\hline Holanda & 1 & 7,69 \\
\hline Itália & 2 & 15,39 \\
\hline Gales & 1 & 7,69 \\
\hline EUA & 3 & 23,08 \\
\hline \multicolumn{3}{|l|}{ Periódicos } \\
\hline Journal of Applied Psychology & 1 & 7,69 \\
\hline Elsevier & 1 & 7,69 \\
\hline Journal of Environmental Research and Public Health & 3 & 23,08 \\
\hline Journals permissions & 1 & 7,69 \\
\hline Oxford University Press & 1 & 7,69 \\
\hline ISTSS & 2 & 15,39 \\
\hline Frontiers in Psychology & 1 & 7,69 \\
\hline Suicide and Life Threatening Behavior & 1 & 7,69 \\
\hline Wiley Online Library & 1 & 7,69 \\
\hline Springer Plus & 1 & 7,69 \\
\hline \multicolumn{3}{|l|}{ Delineamento de pesquisa } \\
\hline Estudo Transversal & 12 & 92,0 \\
\hline Estudo Caso Controle & 1 & 8,0 \\
\hline \multicolumn{3}{|l|}{ Classificação da evidência } \\
\hline Seis & 12 & 92,00 \\
\hline Quatro & 1 & 8,0 \\
\hline
\end{tabular}

Legenda: \% = percentual; $\mathrm{N}=$ número.

Fonte: Elaboração própria (2020).

Em sua maioria os estudos avaliaram os fatores de risco associados à ansiedade e à depressão na atividade militar dos policiais, examinando a frequência, gravidade e a vulnerabilidade dos oficiais no local de trabalho. Tais fatores foram 
considerados como possíveis influenciadores em seu desempenho no trabalho, deixando-os insatisfeitos com sua rotina, em diferentes setores do ambiente de trabalho, como mostra o quadro 2.

\section{Caracterização dos estudos analisados}

Treze estudos fizeram a caracterização sociodemográfica que envolviam policiais com quadro de ansiedade/depressão. A idade média dos policiais militares que relataram sofrer por ansiedade/depressão variou de 19 a 50 anos de idade (Renden, Nieuwenhuys, Savelsbergh, Oudejans, 2015; Giessing et al., 2019; Sherwood et al., 2019). Já em relação ao sexo, os autores que apresentaram dados estatísticos mostraram que além das mulheres serem minoria no âmbito da atividade militar, as mesmas apresentam maior prevalência e são mais vulneráveis a estressores (Renden et al., 2015; Acquadro Maran, Varetto, Zedda, \& Ieraci, 2015).

Quanto à descrição dos fatores que condicionam o surgimento de quadros de ansiedade/depressão entre os policiais militares, foi identificado que os policiais dos serviços operacionais (PSO) e processos operacionais burocráticos são menos propensos a desenvolver transtornos de ansiedade ou um quadro patológico de depressão, isto comparado aos policiais de patrulha (PP), que mediante à demanda de ocorrências se tornam mais vulneráveis e expostos (Mccarthy, Trougakos, \& Cheng, 2016; Maran, Zedda, \& Varetto 2018; Giessing et al., 2019; Cerel, Jones, Brown, Weisenhorn e Patel, 2018; Wickramasinghe, Wijesinghe, Dharmaratne, \& Agampodi, 2016).

No entanto, sobre a forma do quadro de depressão/ansiedade afetar diretamente na qualidade de vida desses policiais, o posicionamento de alguns autores é que quando um oficial está envolvido em cenário violento com potencial para danos graves ou vida direta ameaçada, como emboscada e tiroteio, o risco de TEPT aumenta com a gravidade dos sintomas (Sherwood et al., 2019). Isso se dá, pois a convivência com a violência, o constante risco de morte e as cargas excessivas de trabalho são fatores que causam estresse, ansiedade e podem desencadear a depressão, afetando de forma significativa a qualidade de vida desses policiais (Chopko, Palmieri, \& Adams 2015; Wu, Liu, Li, Tian, \& Tan, 2019; Price, 2017). 
Quadro 2. Publicações incluídas segundo o título do artigo, autor, objetivo principal e perfil amostral e principais resultados. Caxias, MA, Brasil, 2020. (N=13)

\begin{tabular}{|c|c|c|c|c|}
\hline $\begin{array}{l}\mathrm{N}^{\circ} \mathrm{DE} \\
\text { ORDEM } \\
\text { E BASE }\end{array}$ & TÍTULO DO ARTIGO & AUTOR/ ANO & OBJETIVO PRINCIPAL & PERFIL AMOSTRAL \\
\hline $\begin{array}{c}1 \\
\text { BIREME }\end{array}$ & $\begin{array}{l}\text { Organizational and Occupational } \\
\text { Stressors, Their Consequences and } \\
\text { Coping Strategies: A } \\
\text { Questionnaire Survey among } \\
\text { Italian Patrol Police Officers } \\
\end{array}$ & $\begin{array}{l}\text { Maran et al. } \\
\quad(2018)\end{array}$ & $\begin{array}{l}\text { Investigar estressores operacional } \\
\text { organizacionais, suas consequências também em } \\
\text { termos de ansiedade e as estratégias de } \\
\text { enfrentamento } \\
\text { adotadas. }\end{array}$ & $\begin{array}{l}\text { Os participantes foram } 266 \text { Policiais de Patrulha ao } \mathrm{Ar} \\
\text { Livre e } 214 \text { policiais de patrulha. Os Policiais de } \\
\text { Patrulha ao Ar Livre foram } 41,8 \% \text { do total de patrulhas } \\
\text { oficiais do Serviço Operacional. }\end{array}$ \\
\hline $\begin{array}{c}2 \\
\text { BIREME }\end{array}$ & $\begin{array}{l}\text { Are Anxious Workers Less } \\
\text { Productive Workers? It Depends } \\
\text { on the Quality of Social Exchange }\end{array}$ & $\begin{array}{l}\text { Mccarthy et al. } \\
\text { (2016) }\end{array}$ & $\begin{array}{l}\text { Avaliar a exaustão emocional mediado o elo entre } \\
\text { ansiedade no local de trabalho e desempenho do } \\
\text { trabalho, além do efeito cognitivo Interferência. }\end{array}$ & $\begin{array}{l}\text { Os participantes incluíram policiais, seus supervisores, e } \\
\text { seus pares. Um total de } 770 \text { indivíduos responderam à } \\
\text { primeira pesquisa, e } 595 \text { os indivíduos responderam à } \\
\text { segunda pesquisa. }\end{array}$ \\
\hline $\begin{array}{c}3 \\
\text { BIREME }\end{array}$ & $\begin{array}{l}\text { Occupational stress, coping and } \\
\text { mental health in Jamaican police } \\
\text { officers }\end{array}$ & $\begin{array}{l}\text { Nelson e Smith } \\
\quad(2016)\end{array}$ & $\begin{array}{l}\text { Examinar a relação entre as características do } \\
\text { trabalho, enfrentamento e saúde mental de policiais } \\
\text { na Jamaica e para testar se as características do } \\
\text { trabalho estão indiretamente associadas à saúde } \\
\text { mental resultados através do estresse percebido do } \\
\text { trabalho e satisfação no trabalho. }\end{array}$ & $\begin{array}{l}\text { Foi usado um questionário distribuído para } 142 \text { policiais } \\
\text { do Serviço de Polícia Jamaicano, incluiu medidas } \\
\text { demográficas detalhes, características de trabalho, } \\
\text { enfrentamento, estresse percebido, trabalho satisfação e } \\
\text { resultados de saúde mental. }\end{array}$ \\
\hline $\begin{array}{c}4 \\
\text { BIREME }\end{array}$ & $\begin{array}{l}\text { Dutch police officers' preparation } \\
\text { and performance of their arrest } \\
\text { and self-defence skills: A } \\
\text { questionnaire study }\end{array}$ & $\begin{array}{l}\text { Renden et al. } \\
\quad(2015)\end{array}$ & $\begin{array}{l}\text { Avaliar mais detalhes sobre as relações entre a } \\
\text { polícia e experiência dos oficiais, ansiedade e } \\
\text { desempenho em diferentes habilidades de prisão e } \\
\text { autodefesa. }\end{array}$ & $\begin{array}{l}\text { Os entrevistados (oficiais) receberam um questionário } \\
\text { online. No total, } 922 \text { policiais ( } 742 \text { homens, } 180 \\
\text { mulheres), com idade média de } 41,4 \text { anos e experiência } \\
\text { média de trabalho de } 17,9 \text { anos, completou o } \\
\text { questionário. }\end{array}$ \\
\hline $\begin{array}{c}5 \\
\text { BIREME }\end{array}$ & $\begin{array}{l}\text { Occupational stress, anxiety and } \\
\text { coping strategies in police officers }\end{array}$ & $\begin{array}{l}\text { Maran et al. } \\
\quad(2015)\end{array}$ & $\begin{array}{l}\text { Medir o nível de estresse experimentado, as } \\
\text { consequências em termos de ansiedade e } \\
\text { enfrentamento estratégias adotadas em uma amostra } \\
\text { de policiais trabalhando em uma grande cidade no } \\
\text { norte da Itália. }\end{array}$ & $\begin{array}{l}\text { Usaram o Questionário Estresse Policial e o Termômetro } \\
\text { de Angústia para medir estresse ocupacional, o } \\
\text { Inventário de Ansiedade Estado-Traço para medir a } \\
\text { ansiedade e o breve questionário COPE para medir } \\
\text { estratégias de enfrentamento. }\end{array}$ \\
\hline $\begin{array}{c}6 \\
\text { CINAHL }\end{array}$ & $\begin{array}{l}\text { Critical Incident History } \\
\text { Questionnaire Replication: } \\
\text { Frequency and severity of trauma } \\
\text { exposure among officers from } \\
\text { small and midsize police agencies. }\end{array}$ & $\begin{array}{l}\text { Chopko et al. } \\
\text { (2015) }\end{array}$ & $\begin{array}{l}\text { Replicar aFrequência CIHQ e descobertas de } \\
\text { gravidade com uma amostra de PDs e agencia } \\
\text { menores que policiam áreas rurais. }\end{array}$ & $\begin{array}{l}\text { Os participantes eram } 193 \text { policiais da ativa, chamada em } \\
\text { três pequenos grupos para PDs da cidade de médio porte } \\
\text { e no início do treinamento avançado. }\end{array}$ \\
\hline $\begin{array}{c}7 \\
\text { PUBMED }\end{array}$ & $\begin{array}{l}\text { Assessing the Relative Impact of } \\
\text { Diverse Stressors among Public } \\
\text { Safety Personnel }\end{array}$ & $\begin{array}{l}\text { Carleton et al. } \\
\text { (2020) }\end{array}$ & $\begin{array}{l}\text { Evidenciar que os estressores } \\
\text { estressonizacionais, } \\
\text { significativamente associados a sintomas de } \\
\text { estresse pós-traumático. }\end{array}$ & $\begin{array}{l}\text { Os participantes foram recrutados través de e-mails } \\
\text { enviados para PSP de trabalho ativo, incluindo membros } \\
\text { civis que trabalham para a polícia e bombeiros } \\
\text { voluntários. }\end{array}$ \\
\hline
\end{tabular}




\begin{tabular}{|c|c|c|c|c|}
\hline $\begin{array}{c}8 \\
\text { PUBMED }\end{array}$ & $\begin{array}{l}\text { Traits and Psychophysiological } \\
\text { Stress } \\
\text { Responses on Police Recruits' } \\
\text { Shooting Behavior in Reality- } \\
\text { Based Scenarios }\end{array}$ & $\begin{array}{l}\text { Giessing et al. } \\
\quad(2019)\end{array}$ & $\begin{array}{l}\text { Examinar o stress psicológico, fisiológico e } \\
\text { comportamental respostas dos recrutas policiais em } \\
\text { cenários baseados na realidade. }\end{array}$ & $\begin{array}{l}3 \text { mulheres, } 16 \text { homens participaram durante duas } \\
\text { sessões de treinamento. Os participantes foram entre } 19 \text { e } \\
33 \text { anos de idade. Todos os recrutas já estão de serviço } \\
\text { há } 2 \text { meses. }\end{array}$ \\
\hline $\begin{array}{c}9 \\
\text { PUBMED }\end{array}$ & $\begin{array}{l}\text { Health-Related Quality of Life } \\
\text { and Its Determinants among } \\
\text { Criminal Police Officers }\end{array}$ & Wu et al. (2019) & $\begin{array}{l}\text { Examinar o estado de saúde dos oficiais da polícia } \\
\text { criminal na China, utilizando a EuroQol five- } \\
\text { dimension three-level (EQ-5D-3L) como medida da } \\
\text { HRQoL e para explorar os fatores determinantes da } \\
\text { HRQoL nos oficiais da polícia criminal em } \\
\text { comparação com a população adulta em geral. }\end{array}$ & $\begin{array}{l}\text { Entrevistados } 300 \text { oficiais, para coletar sintomas de } \\
\text { HRQ, ansiedade e depressão, informaçóes } \\
\text { sociodemográficas, relacionadas à aúde } \\
\text { comportamentos, condições sociais e de trabalho e } \\
\text { histórico de doenças crônicas. }\end{array}$ \\
\hline $\begin{array}{c}10 \\
\text { PUBMED }\end{array}$ & $\begin{array}{l}\text { Factors for Adverse Psychological } \\
\text { Outcomes Among Police Officers: } \\
\text { A Systematic Literature Review }\end{array}$ & $\begin{array}{l}\text { Sherwood et al. } \\
\text { (2019) }\end{array}$ & $\begin{array}{l}\text { Visar investigar sistematicamente a literatura } \\
\text { relevante em torno dos vários domínios de risco e } \\
\text { suas associações com a saúde mental adversa mais } \\
\text { comum desfechos estudados: depressão, ansiedade, } \\
\text { TEPT e Burnout. }\end{array}$ & $\begin{array}{l}\text { A estratégia para esta revisão foi em artigos que } \\
\text { contenham os termos de pesquisa no título, abstrato, ou } \\
\text { como uma palavra-chave ou título principal. }\end{array}$ \\
\hline $\begin{array}{c}11 \\
\text { PUBMED }\end{array}$ & $\begin{array}{l}\text { Suicide Exposure in Law } \\
\text { Enforcement Officers }\end{array}$ & Cerel et al. (2018) & $\begin{array}{l}\text { Determinar a prevalência de exposição ao suicídio } \\
\text { ocupacional e pessoal em oficiais de execução da } \\
\text { lei (LEOs). }\end{array}$ & $\begin{array}{l}\text { Foi realizada uma pesquisa online com } 813 \text { policiais e } \\
\text { responderam sobre sua exposição ao suicídio, se as cenas } \\
\text { ficaram com eles e os sintomas atuais de depressão, } \\
\text { ansiedade, TEPT e ideação suicida. }\end{array}$ \\
\hline $\begin{array}{c}12 \\
\text { PUBMED }\end{array}$ & $\begin{array}{l}\text { Psychiatric Disability in Law } \\
\text { Enforcement Officers }\end{array}$ & Price (2017) & $\begin{array}{l}\text { Analisar se o estresse ambiental tem se mostrado } \\
\text { um fator principal para determinar se os oficiais vão } \\
\text { desenvolver prejuízo à saúde mental após exposição } \\
\text { a um incidente crítico }\end{array}$ & $\begin{array}{l}\text { A amostra foi feita com polícia expostos a um incidente } \\
\text { critico (morte, suicídio, ou ferimentos graves de um } \\
\text { colega de trabalho, um tiroteio em serviço, homicídios de } \\
\text { alguém da família }\end{array}$ \\
\hline $\begin{array}{c}13 \\
\text { PUBMED }\end{array}$ & $\begin{array}{l}\text { The prevalence and associated } \\
\text { factors of depression in policing: a } \\
\text { cross sectional study in Sri Lanka }\end{array}$ & $\begin{array}{l}\text { Wickramasinghe } \\
\text { et al. (2016) }\end{array}$ & $\begin{array}{l}\text { Determinar a prevalência e os fatores associados } \\
\text { depressão entre policiais da divisão policial de } \\
\text { Kandy, Sri Lanka. }\end{array}$ & $\begin{array}{l}\text { Utilizando uma amostra aleatória simples de } 750 \\
\text { policiais empregados na divisão policial de Kandy, Sri } \\
\text { Lanka. Um questionário auto-aplicado, incluindo "Escala } \\
\text { de Depressão peradeniya" para avaliar a depressão, foi } \\
\text { utilizado para coletar dados. }\end{array}$ \\
\hline
\end{tabular}

Fonte: Dados da pesquisa (2020). 


\section{Discussão}

\section{Caracterização sociodemográfica de policiais militares com ansiedade/depressão}

As evidências na literatura revelam que os policiais apresentam um risco maior de desenvolverem um ou mais sintomas relacionados à saúde mental do que a população em geral no exercício de suas atividades militares, tendo em vista a sua jornada de trabalho muitas vezes estressante, com riscos físicos e mentais, afeta a vida pessoal e familiar desses profissionais e favorece o declínio de sua qualidade de vida, o que corrobora com os estudos da maioria dos autores citados em virtude da exposição e vulnerabilidade frente ao cenário de perigo iminente (Castro, Rocha, \& Cruz, 2020; Grossi Filho, 2019).

Quando se consideram as características sociodemográficas da amostra do estudo em relação ao sexo, policiais militares do sexo feminino apresentam nível mais elevado, assim como referem mais sintomas psicológicos do que físicos. Elas também relatam considerar que a estabilidade no emprego e não o prazer advindo do trabalho compensa o estresse vivenciado em decorrência dele (Bezerra, Minayo, \& Constantino, 2013). Tais dados evidenciam de forma fundamentada que em grande parte as pesquisas revelam que os policiais do sexo feminino são mais vulneráveis a estressores organizacionais.

Para as mulheres policiais, essa jornada é considerada mais exaustiva, pois além da carga horária de trabalho habitual, há ainda a realização de atividades no âmbito familiar, relacionadas aos cuidados da casa e dos filhos, o que influencia no aparecimento de picos de estresse mais intensos em função da diferença de gênero, além de, muitas vezes, alterações hormonais. Tudo isso, associado à demanda de trabalho, se torna um fator prejudicial à saúde mental (Capelle \& Melo, 2010).

\section{Descrição dos fatores que condicionam o surgimento de ansiedade/depressão entre os policiais militares}

No estudo de Mendes (2017), a prevalência de ansiedade se apresenta em oficiais com tempo de serviço de 13 anos em média. Isso demonstra que, quanto mais tempo os participantes são expostos a cenário de alto estresse e situações de risco, mais se tornam susceptíveis a sofrer de ansiedade; assim, os traços de ansiedade tendem a crescer conforme seja maior o tempo de serviço, afetando o seu desempenho de trabalho e deixando os profissionais insatisfeitos. Tais informações divergem em parte do estudo de Renden et al. (2015), apenas quanto ao tempo em média de serviço dos policiais, sendo 17 anos; contudo, apresentam dados similares quanto às queixas e insatisfação profissional em função de transtornos que afetam sua saúde emocional.

O documento "Doenças relacionadas ao trabalho: manual de procedimentos para os serviços de saúde", do Ministério da Saúde e vários outros estudos apontam que, na população em geral, as taxas de acometimento estimadas de Transtorno de Estresse Pós-Traumático (TEPT) são de 1 a 3\%, podendo aumentar para 5 a 15\%, se forem consideradas formas subclínicas da doença. Nos Estados Unidos, um estudo epidemiológico estimou a prevalência de TEPT em 7,8\% na população geral, distribuídos em 5,0\% dos homens e 10,4\% das mulheres. Os policiais militares se mostraram com elevada prevalência de TEPT (16,7\%) quando comparados à população em geral (Moura, 2013).

Vancini et al. (2018) demonstraram a classificação de ansiedade e depressão em policiais de acordo com a rotina de trabalho. Segundo os resultados, 67,6\% e 20,5\% dos policiais relataram médio e altos níveis de ansiedade, respectivamente. Para classificação de depressão, 14,7\% leve a moderada, e 8,8\% moderada a níveis de depressão severos. Caracterizando o perfil da ansiedade e depressão, o que torna as chances maiores de sofrer de um ou mais transtorno de acordo com seu trabalho, os policiais de setores operacionais são menos propensos a serem acometidos, pois têm menos contato com estresse excessivo e exaustivo, o qual pode levar a distúrbios psicológicos e impactar negativamente a saúde mental e, consequentemente, o desempenho profissional. Corroborando com os estudos de Carleton et al. (2020), evidencia-se que os policiais são mais vulneráveis a ter mais de um transtorno. 
Research, Society and Development, v. 10, n. 10, e201101018702, 2021

(CC BY 4.0) | ISSN 2525-3409 | DOI: http://dx.doi.org/10.33448/rsd-v10i10.18702

A profissão de Policial Militar é um exemplo de atividade laboral que por si só traz aos seus colaboradores algumas mudanças no seu estado psíquico e emocional, por ser uma instituição que preza pela extrema ordem e respeito, com um treinamento rígido aos ingressantes e com uma exigência intensa ao cumprimento das regras institucionalizadas, que são baseadas em dois pilares: disciplina e hierarquia. Mesmo apesar de garantir a segurança da população em meio às mais variadas ocorrências da profissão, ainda se tornam mais suscetíveis e predispostos a transtornos de ansiedade e quadros depressivos (Paulino \& Lourinho, 2014).

O medo de ser reconhecido como agente de segurança durante as folgas, de ser morto ou agredido no desempenho de suas funções, provoca uma vitimização e um constante estado de alerta nos policiais, tanto para eles próprios quanto para seus familiares. Essa forma de defesa permanece frequente na mente do profissional e afeta diretamente várias áreas de sua vida, principalmente o convívio social, pois se priva de muitas atividades ou de frequentar inúmeros locais para prevenir que algum malefício aconteça com sua integridade física, moral e emocional (Souza et al., 2012).

Segundo Alcântara, Sampaio, Assunção e Silva (2014), que vai ao encontro do estudo de Maran et al. (2018), ambientes de trabalho com alta demanda profissional exercem efeito direto na capacidade para o trabalho. Além do efeito direto na capacidade para o trabalho, essas características também apresentam um efeito indireto por meio da sua influência no estado geral de saúde do trabalhador, pois os policiais do setor administrativo e processos operacionais internos são menos acometidos por ansiedade/depressão do que os policiais de patrulha externa.

Reis e Faiad (2014), por sua vez, têm como estudo de sua pesquisa a instabilidade emocional como fator geral para apresentar características compatíveis com o aumento de sintomas de depressão, ansiedade, irritabilidade e baixa estabilidade emocional. Segundo tal estudo, esses profissionais apresentam sentimentos de desesperança, tristeza, baixa autoestima, desânimo e dificuldade em lidar com situações de auto exposição, o que torna provável a perda de controle, bem como o agir sem prévia reflexão, resultando por vezes no uso da força em momentos da abordagem, o que pode levar ao envolvimento em problemas.

Estudos envolvendo policiais apresentaram elevada prevalência de transtornos mentais (sintomas psicossomáticos, depressão e ansiedade) (Minayo, Assis, \& Oliveira, 2011) e ideação suicida (Stanley, Hom, \& Joiner, 2016) nessa classe profissional. Outros trabalhos, por sua vez, identificaram altas taxas de suicídios entre os ocupantes das carreiras policiais, em grande parte decorrente da presença de transtornos mentais (principalmente depressão) e das características internas que envolvem a profissão (elevada exposição a traumas físicos e emocionais) (Stanley et al., 2016).

Em uma coorte retrospectiva realizada com dados de 31.110 policiais militares entre os anos de 2006 a 2016 no Sul do Brasil, com média de idade no final do tempo de acompanhamento de 41,4 \pm 9,2 anos (mínima de 20 anos, máxima de 85 anos), foram notificados 650 óbitos no período do estudo, com 43 relacionadas a suicídios (6,6\% do total de óbitos), demostrando que os atuantes na segurança pública estão em alto risco de suicídio. Especialmente entre as mulheres, com idade prevalente de 44 anos, relacionadas ao trabalho da polícia militar como pretexto de diminuir o medo da morte, tolerância a dor física, como fator principal causando o progresso da ideação para tentativas. Todos esses fatores somam-se ainda à questão da exposição ao combate durante o trabalho do policial (Gomes, De Araújo, \& Gomes, 2018).

\section{A forma como o quadro de ansiedade/depressão afeta a qualidade de vida dos policiais}

Nos achados relacionados à qualidade de vida diante de um quadro com ansiedade/depressão, os autores relatam que quando um oficial está envolvido em cenário violento com potencial para danos graves ou vida direta ameaçada, a qualidade de vida desse oficial, assim como sua saúde, são comprometidas. Tais fatos corroboram um estudo com 506 policiais no estado de São Paulo, o qual utilizou um questionário que relata o trabalho do policial como desgastante, estando diretamente ligado ao 
estresse, cansaço, sofrimento psíquico, e que mostra que a qualidade de vida desses profissionais está propriamente ligada a fatores sociais e psicológicos (Arroyo, Borges, \& Lourenção, 2019).

Segundo Pinto, Figueiredo e Souza (2013), que estudaram as condições de trabalho, saúde e qualidade de vida de 914 policiais civis do Rio de Janeiro, ocorreu uma prevalência de transtornos mentais comuns em 21\% dos participantes. O instrumento de rastreio empregado foi o SRQ-20. Aparentemente, a carga de trabalho e o tempo de exposição ao estresse apresentam relação direta com o risco de doença, pois diante desses cenários o policial acaba se tornando um público alvo em comparação à população em geral.

Wagner, Stankievich e Pedroso (2013), por sua vez identificaram que policiais civis com mais de 10 anos na profissão apresentaram saúde mental e qualidade de vida mais deterioradas do que seus colegas com menor tempo de profissão. O que torna de fundamental importância a adoção de estratégias mais dinâmicas de supervisão à saúde desses profissionais com equipes especializadas em conscientização e saúde mental, para que se possa otimizar o ambiente de trabalho.

Segundo Coelho-Alves, Bendassolli e Guedes-Godim (2017), as emoções negativas e positivas podem ocasionar uma exaustão emocional, decorrente do trabalho corriqueiro, que leva a uma insatisfação do trabalho do profissional. Indicando que os policiais se encontram em um nível alto de exaustão emocional, os policiais que atuam externamente apresentam maiores indicadores de exaustão emocional do que seus colegas atuando internamente. Quanto aos fatores do trabalho emocional, quanto mais diversos e intensos os afetos vivenciados pelos policiais militares, maior a tendência de exaustão emocional, o que está fortemente associado a indicadores de prejuízos ao bem-estar.

Segundo Lima, Andrade, Borba, Barbosa e Brito (2018), em seus estudos com policiais militares, a qualidade de vida dos mesmos apresentou-se mais elevada entre aqueles participantes integrantes do Batalhão de Choque, os quais demonstraram um nível menor de estresse em comparação com os demais, o que poderia ser esclarecido pelo fato de a companhia ser uma unidade militar treinada para situações especiais de extrema tensão. Tais fatos corroboram o estudo de Sherwood et al. (2019), demostrando a importância do preparo físico e mental do profissional que irá atuar em situações de estresse, com risco iminente à vida, tendo o controle desses cenários e, logo, proporcionando segurança na sua atuação profissional, o indivíduo apresenta melhores respostas a tais situações.

\section{Considerações Finais}

Os treze estudos abordados possibilitaram evidenciar que a profissão dos policiais militares é estressante, exaustiva, com risco à saúde física e mental dos atuantes e, consequentemente, acaba gerando sintomas de ansiedade e depressão que afetam o desempenho do profissional na área, trazendo insatisfação nos seus serviços e atingindo também a qualidade de vida desses policiais. O sexo feminino foi o que mais se destacou na apresentação dos sintomas para ansiedade/depressão, com idade entre 19 a 50 anos, com 17 anos de serviço em média. Além disso, o setor de serviços operacionais se mostrou mais exaustivo, tornando aqueles nele inseridos mais vulneráveis, uma vez que acabam por ter mais contato com estressores.

Durante a elaboração do estudo, o tema exposto teve limitações, como na estratégia de busca que foi alterada para ampliar a busca por artigos relacionados e que respondiam à problemática do tema, pois o número de estudos encontrados era pequeno ou apresentava baixo nível de evidências. Consequentemente, mais estudos devem ser realizados na área com os policias militares, atualizando os estudos desse público alvo.

Os resultados deste estudo mostram, diante das apresentações dos conhecimentos dos sintomas mais frequentes encontrados, a importância da promoção e prevenção da saúde mental no trabalho dos policiais, pretendendo torná-los menos predispostos e vulneráveis quanto aos transtornos ansiosos e depressivos, isso por meio do reconhecimento dos fatores de risco 
Research, Society and Development, v. 10, n. 10, e201101018702, 2021

(CC BY 4.0) | ISSN 2525-3409 | DOI: http://dx.doi.org/10.33448/rsd-v10i10.18702

frente aos cenários de alto risco e estresse em seu cotidiano. Além disso, é possível otimizar o ambiente de trabalho através da conscientização, promovendo conhecimento adequado sobre transtornos emocionais que são rotineiros e tornando possível uma redução nos índices de adoecimento dessa categoria. Assim, consequentemente, resultados mais eficazes no combate a esses transtornos de origem emocional podem ser alcançados.

Por fim, na atuação do enfermeiro frente a esses pacientes, o primordial a ser feito é a promoção e prevenção da saúde, para fazer com que esse público procure mais o atendimento especializado e adquira conhecimento mesmo que superficial para reconhecer os sintomas, possibilitando uma redução nos índices de adoecimento dessa categoria e gerando, consequentemente, resultados mais eficazes.

\section{Referências}

Acquadro Maran, D., Varetto, A., Zedda, M., \& Ieraci, V. (2015). Occupational stress, anxiety and coping strategies in police officers. Occupational Medicine, 65(6), 466-473.

Alcântara, M. A., Sampaio, R. F., Assunção, A. Á., \& Silva, F. C. M. (2014). Work Ability: using structural equation modeling to assess the effects of aging, health and work on the population of Brazilian municipal employees. A Journal of Prevention, Assessment \& Rehabilitation, 49(3), 465-472.

Apóstolo, J. L. A., Figueiredo, M. H., Mendes, A. C., Rodrigues, M. A. (2011). Depression, anxiety and stress in primary health care users. Rev. Latino-Am. Enfermagem, 19(2), 348-353.

Arroyo, T. R., \& Borges, M. A., \& Lourenção, L.G. (2019). Saúde e qualidade de vida de policiais militares. Rev Bras Promoç Saúde, 32 , e7738.

Bezerra, C. M., Minayo, M. C. S., \& Constantino, P. (2013). Estresse ocupacional em mulheres policiais. Ciênc. saúde coletiva, 18(3), 657-666.

Capelle, M. C. A., \& Melo, M. C. O. L. (2010). Mulheres policiais, relações de poder e de gênero na polícia militar de Minas Gerais. Ram, Rev. Adm. Mackenzie, 11(3), 71-99.

Carleton, R. N., Afifi, T. O., Taillieu, T., Turner, S., Mason, J. E., Ricciardelli, R., \& Krakauer, R. L. (2020). Assessing the Relative Impact of Diverse Stressors among Public Safety Personnel. Int J Environ Res Public Health, 7(4), e1234.

Castro, M. C., Rocha, R., \& Cruz, R. (2019). Mental health of the brazilian police policy: theoretical-methodological trends. Psic., Saúde \& Doenças, 20(2), $525-541$.

Cerel, J., Jones, B., Brown, M., Weisenhorn, D. A., Patel, K. (2018). Suicide Exposure in Law Enforcement Officers. Suicide And Life-threatening Behavior, 49(5), 1281-1289.

Chopko, B. A., Palmieri, P. A., \& Adams, R. E. (2015). Critical incident history questionnaire replication: frequency and severity of trauma exposure among officers from small and midsize police agencies. Journal of Traumatic Stress, [28(2), 157-161.

Coelho-Alves, J. S., Bendassolli, P. F., \& Guedes-Gondim, S. M. (2017). Trabalho emocional e burnout: um estudo com policiais militares. Av. Psicol. Latinoam., 35(3), 459-472.

Ercole, F. F., Melo, L. S., \& Alcoforado, C. L. G. (2014). Integrative review versus systematic review. Revista Mineira de Enfermagem, 18(1), 9-11.

Giessing, L., Frenkel, M. O., Zinner, C., Rummel, J., Nieuwenhuys, A., Kasperk, C., \& Plessner, H. (2016). Effects of Coping-Related Traits and Psychophysiological Stress Responses on Police Recruits' Shooting Behavior in Reality-Based Scenarios. Front Psychol., 10, 1-16.

Gomes, D. A. R., De Araújo, R. M. F., \& Gomes, M. S. (2018). Incidence of suicide among military police officers in South Brazil: An 11-year retrospective cohort study. Comprehensive Psychiatry, 85, 61-66.

Grossi Filho, M. (2019). Indicadores de saúde mental e qualidade de vida nas diferentes carreiras da polícia civil (Dissertação de mestrado). Universidade Federal de Uberlândia, Uberlândia, MG, Brasil.

Guimarães, A. M. V., Silva Neto, A. C., Vilar, A. T. S., Almeida, B. G. C., Fermoseli, A. F. de O., \& Albuquerque, C. M. F. de. (2015). Transtornos de ansiedade: um estudo de prevalência sobre as fobias específicas e a importância da ajuda psicológica. Semina Cienc Biol Saude, 3(1), 115-28.

Lima, S. M. O., Andrade, G. A., Borba, N. V. C., Barbosa, L. N. F., \& Brito, E. C. (2018). Avaliação do nível da síndrome de Burnout e qualidade de vida em policiais militares do estado de Pernambuco (Trabalho de conclusão de curso). Faculdade Pernambucana de Saúde, Recife, PE, Brasil.

Maran, D. A., Zedda, M., \& Varetto, A. Organizational and Occupational Stressors, Their Consequences and Coping Strategies: a questionnaire survey among italian patrol police officers. International Journal Of Environmental Research And Public Health, 15(1), 466-473.

Mccarthy, J. M., Trougakos, J. P., \& Cheng, B. H. (2016). Are Anxious Workers Less Productive Workers? It Depends on the Quality of Social Exchange. Journal of Applied Psychology, 101(2), 279-291. 
Research, Society and Development, v. 10, n. 10, e201101018702, 2021 (CC BY 4.0) | ISSN 2525-3409 | DOI: http://dx.doi.org/10.33448/rsd-v10i10.18702

Mendes, M. M. M. O. (2017). O impacto dos transtornos de ansiedade na saúde dos trabalhadores da polícia militar (Trabalho de Conclusão de Curso). Universidade Federal da Paraíba, João Pessoa, PA, Brasil, 2017.

Minayo, M. C. S., Assis, S. G., \& Oliveira, R. V. C. (2011). Impacto das atividades profissionais na saúde física e mental dos policiais civis e militares do Rio de Janeiro (RJ, Brasil). Ciência \& Saúde Coletiva, 16, 2199-2209.

Moura, G. O. (2013). História de los critérios diagnósticos de trastorno de estrés post-traumático. Anais Congreso Internacional de Investigación Y Práctica Profesional En Psicología Xx Jornadas De Investigación Noveno Encuentro de Investigadores En Psicología Del Mercosur, $5 .$, Buenos Aires, Argentina, 5.

Nelson, K. V., \& Smith, A. P. (2016). Occupational stress, coping and mental health in Jamaican police officers. Occupational Medicine, 66(6), 488-491.

Oliveira, F. B. M., Costa, A. C. A. L., Alves, D. L., França, J. F., Macedo, M. S., \& Santos, R. D. (2016). Relação entre a sobrecarga de trabalho e erros de administração de medicação na assistência hospitalar. Revista Ciências \& Saberes, 2(2), 325-334.

Oliveira, K. L., \& Santos, L. M. (2010). Percepção da saúde mental em policiais militares da força tática e de rua. Sociologias, 12(25), 224-250.

Paulino, F. R., \& Lourinho, L. A. (2014). O adoecimento psicológico do policial militar do Ceará. Revista Trabalho e Sociedade, 2 (2), 58-77.

Pinto, L. W., Figueiredo, A. E. B., \& Souza, E. R. (2013). Sofrimento psíquico em policiais civis do Estado do Rio de Janeiro. Ciência \& Saúde Coletiva, 18, 633-644.

Price, M. (2017). Psychiatric Disability in Law Enforcement Officers. Behav Sci Law., 35(2), 113-123.

Reis, M. S., \& Faiad, C. (2014). Escala de instabilidade emocional para a segurança pública. Psico-USF, 19(1), 87-96.

Renden, P. G., Nieuwenhuys, A., Savelsbergh, G. J. P., \& Oudejans, R. R. D. (2015). Dutch police officers' preparation and performance of their arrest and self-defence skills: a questionnaire study. Applied Ergonomics, 49, 8-17.

Sherwood, L., Hegarty, S., Vallières, F., Hyland, P., Murphy, J., Fitzgerald, G., \& Reid, T. (2019). Identifying the Key Risk Factors for Adverse Psychological Outcomes Among Police Officers: a systematic literature review. Journal Of Traumatic Stress, 32(5), 688-700.

Souza, E. R., Minayo, M. C. S., Silva, J. G., Pires, T. O. (2012). Fatores associados ao sofrimento psíquico de policiais militares da cidade do Rio de Janeiro. Cadernos de Saúde Pública, 28(7), 1297-1311.

Stanley, I. H., Hom, M. A., \& Joiner, T. E. (2016). A systematic review of suicidal thoughts and behaviors among police officers, firefighters, EMTs, and paramedics. Clinical Psychology Review, 44, 25-44.

Vancini, R. L., de Lira, C. A. B., Anceschi, S. A., Rosa, A. V., Lima-Leopoldo, A. P., Leopoldo, A. S., \& Knechtle, B. (2018). Anxiety, depression symptoms, and physical activity levels of eutrophic and excess-weight Brazilian elite police officers: a preliminary study. Psychology research and behavior management, 11, 589-595.

Wagner, L. C., Stankievich, R. A. P., \& Pedroso, F. (2012). Saúde mental e qualidade de vida de policiais civis da região metropolitana de Porto Alegre. Rev Bras Med Trab, 10(2), 64-71.

Wickramasinghe, N. D., Wijesinghe, P. R., Dharmaratne, S. D., \& Agampodi, S. B. (2016). The prevalence and associated factors of depression in policing: a cross sectional study in sri lanka. Springerplus, 5(1), 589-595.

Wu, X., Liu, Q., Li, Q., Tian, Z., \& Tan, H. (2019). Health-Related Quality of Life and Its Determinants among Criminal Police Officers. Int J Environ Res Public Health, 16(8), e1398. 